\title{
Single-electron states in the quantum Hall effect
}

\author{
N.B. Zhitenev ${ }^{\mathrm{a}, *}$, T.A. Fulton ${ }^{\mathrm{a}}$, A. Yacoby ${ }^{\mathrm{b}}$, H. Hess ${ }^{\mathrm{c}}$, L.N. Pfeiffer ${ }^{\mathrm{a}}$, K.W. West ${ }^{\mathrm{a}}$ \\ ${ }^{a}$ Bell Labs, Lucent Technologies, IC-324, 600 Mountain Avenue, Murray Hill, NJ 07974, USA \\ ${ }^{\mathrm{b}}$ Weizmann Institute of Science, Rehovot, Israel \\ ${ }^{\mathrm{c}}$ Phasemetrics Inc., San Diego, CA 92121, USA
}

\begin{abstract}
Spatially localized electronic states in a two-dimensional electron gas in the quantum Hall regime are imaged using a scanning single-electron-transistor probe. Two different regimes of localization are identified depending on the strength of the built-in long-range density fluctuations. In the smoother regions localized states merge into dielectric-like blobs a few $\mu \mathrm{m}$ in extent. In the places with a stronger density gradient complex patterns occur which change markedly with an addition of a single electron. The simplest appearance of the latter is a ring collapsing toward the center as an electron is added to the area. (c) 2000 Elsevier Science B.V. All rights reserved.
\end{abstract}

PACS: $73.40 . \mathrm{Hm} ; 73.23 . \mathrm{Hk} ; 73.20 . \mathrm{Fz}$

Keywords: Quantum Hall effect; Localization; Single-electron transistor; Scanning probe

It is accepted that in the quantum Hall $(\mathrm{QH})$ regime electronic states at the Fermi level in the interior of two-dimensional electron gas (2DEG) are localized $[1,2]$. To advance the physics of localization further, new microscopic characterization tools are desirable. By definition, localization means a confinement of the electron wave function within a restricted area, which may result in an inhomogeneous charge and potential distribution. Recently, several scanning probe experiments [3-6] have demonstrated the capability to image electrical properties in a two-dimensional electron system. In addition to micrometer-scale features showing the Hall potential distribution and edge-state

* Corresponding author. Tel. 908-582-4980; fax. 908-582-7660.

E-mail address: zhiten@lucent.com (N.B. Zhitenev) contours [5,6], finer structures in compressibility and resistance were seen [4] down to the scale of $100 \mathrm{~nm}$. Here, we look for localized states in the $\mathrm{QH}$ regime near filling factor $v=2$, employing the technique of Ref. [6] with higher resolution in position and density. The behavior observed is complex, but contains systematic features. In particular, individual objects are seen which are evidently single-electron localized states.

To measure the local electrostatic potential we employ a single-electron transistor fabricated on a tip of a tapered glass fiber as described in Refs. $[3,6,7]$. The tip is scanned just above the surface of a GaAs/AlGaAs heterostructure having the 2DEG buried $100 \mathrm{~nm}$ below the surface. The average density $n_{0}$ of the $2 \mathrm{DEG}$ is $1.5 \times 10^{11} \mathrm{~cm}^{-2}$. 
Additionally, a bias $V_{\mathrm{g}}$ on the backgate, located $5.4 \mu \mathrm{m}$ under $2 \mathrm{DEG}$, allows us to vary the density of electrons $\left(V_{\mathrm{g}}=-1 \mathrm{~V}\right.$ reduces the density by $\left.1.3 \times 10^{10} \mathrm{~cm}^{-2}\right)$. The SET is operated in a feedback loop maintaining constant potential on the SETs central island. The feedback voltage $V_{\mathrm{fb}}$, applied to the 2DEG and backgate, directly reflects the local potential seen by the SET. In addition, we modulate the 2DEG density at a frequency above the roll-off of the feedback loop and record the "transparency", the AC potential seen by the SET. With all other charges being immobile at low temperature, the contrast in the AC signal is determined solely by charges in the 2DEG layer. Transparency is only seen near integer filling factors, and denotes low electron compressibility and/or high resistivity. All measurements shown are carried out at temperature of $0.8 \mathrm{~K}$.

In the experiments, the magnetic field is set at $\sim 3.1 \mathrm{~T}$, nominally corresponding to $v=2$, although the local densities vary by a few percent. At a variety of arbitrary locations we then record either the $V_{\mathrm{g}}$ (density) dependence of the $\mathrm{DC}\left(-V_{\mathrm{fb}}\right)$ and $\mathrm{AC}$ (transparency) signals at fixed locations, or spatial images of these at a set of fixed $V_{\mathrm{g}}$.

For the density dependence, the SET tip is fixed in place and the 2DEG density is swept so that the local filling factor passes through the integer value. Broadly, the behavior tends to be of one of two types. Typical records of these responses are shown in Fig. 1a. In both, the DC potentials $\left(-V_{\mathrm{fb}}\right)$ display the same characteristic step of $\sim h \omega_{\mathrm{c}} / 2 \pi$ associated with the Fermi level passing between the third and second Landau levels $[6,8]$, but the accompanying AC signal amplitude differs by up to $\times 10$. Quantitatively, the smaller signals in the right panel in Fig. 1a correspond to the derivative of the DC step, as expected at equilibrium (the structure in the signal is discussed below). The larger size of the signal in the leftmost AC curve shows that a significant resistance is impeding charge transport to the particular location at the frequency of $70 \mathrm{~Hz}$. Correspondingly, an out-of-phase component of the AC signal appears at the condition where the charging rate of the location is comparable with the AC modulation rate. In the following, we examine some typical spatial structures of regions first without and then with $\mathrm{AC}$ equilibrium charging.

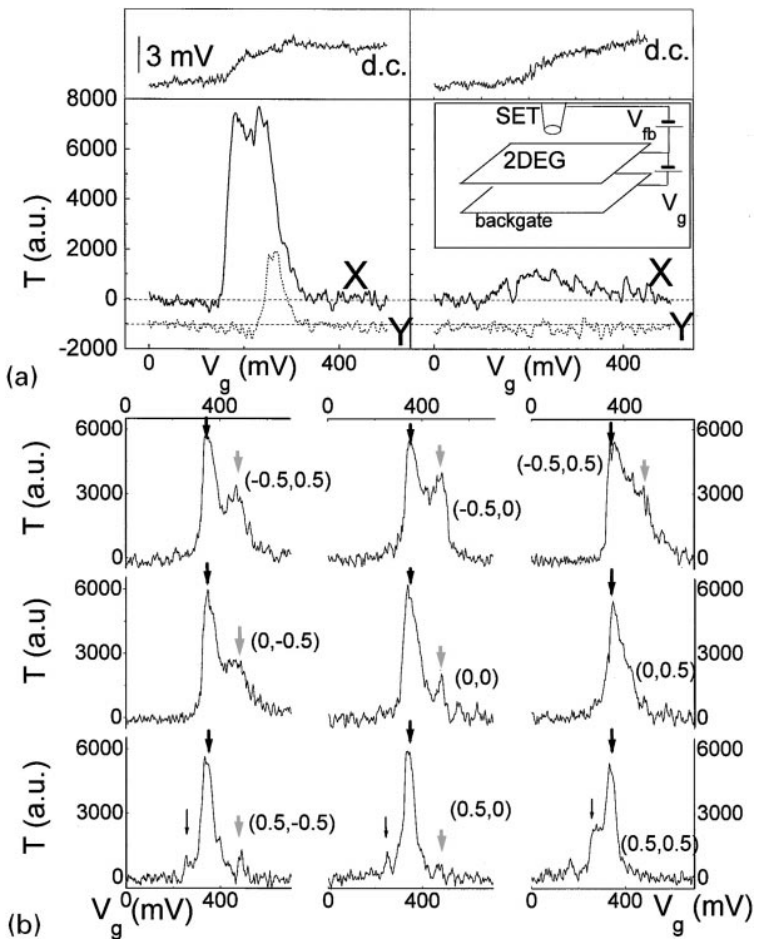

Fig. 1. (a) Two typical sets (left and right panels) of data acquired by SET as the electron density $\left(\sim V_{\mathrm{g}}\right)$ is swept through $v=2$ at different spatial locations. On each panel: top - DC signal displaying the characteristic step of the chemical potential as the Fermi level moves from second to third Landau level; bottom in-phase component (X) and out-of-phase component (Y) (shifted down by 1000) of AC transparency signal. Inset: Sketch of the measurement setup. (b) In-phase AC transparency signals as a function of $V_{g}$ measured at nine spatial locations arranged in $3 \times 3$ grid over an area of strong transparency of $1 \times 1 \mu \mathrm{m}^{2}$. Different types of arrows mark the equivalent peaks detected at distinct spatial locations.

A set of in-phase AC transparency data taken in an area with incomplete charging is shown in Fig. 1b. A region of $\sim 1 \times 1 \mu \mathrm{m}^{2}$ is sampled on a $3 \times 3$ square grid. The maximum shift of the peaks across the area is $\sim 100 \mathrm{mV}$ of $V_{\mathrm{g}}$, for an typical local density gradient of $<1 \times 10^{13} \mathrm{~cm}^{-3}$. Such a gradient is representative for the smoother areas in the sample, and is generally associated with non-equilibrium charging. As seen from Fig. 1b, a typical AC curve is not smooth but rather appears to be a superposition of smaller peaks centered at different $V_{\mathrm{g}}$ voltages. The characteristic spatial cor- 


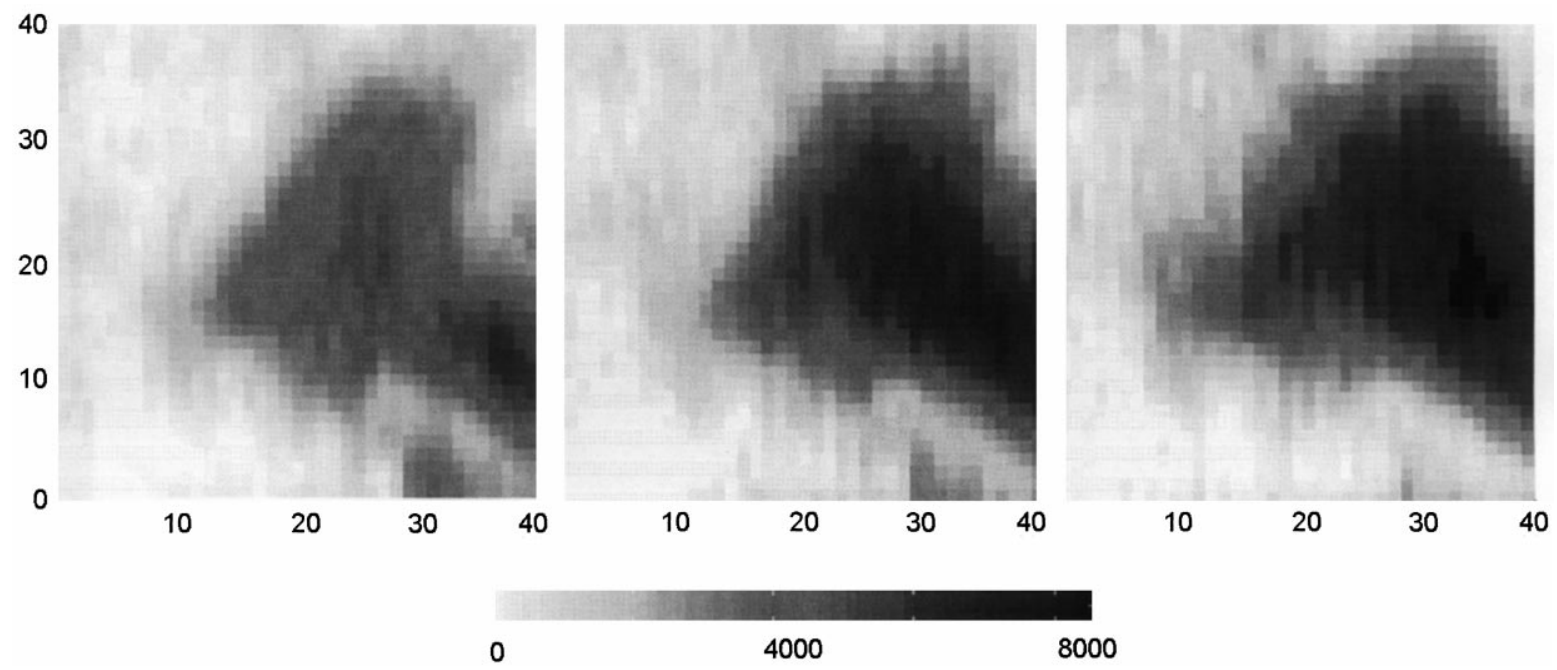

Fig. 2. Spatial images of in- phase AC signal over $5 \times 5 \mu \mathrm{m}^{2}$ area of strong transparency taken at three different densities. $V_{\mathrm{g}}$ is changed by $10 \mathrm{mV}$ between images.

relation length is in the micrometer range. As the strength of a particular peak changes with spatial position, the overall maximum changes its shape and slides across the $V_{\mathrm{g}}$ scale. Apparently, each such peak is an event centered around a distinct location and density.

Typically, the evolution of strong transparency passes through two stages. First, as the density is changed toward the local integer $v$, a number of neighboring spots with typical individual diameters of $\sim 0.5 \mu \mathrm{m}$ become slightly transparent at close but separate values of $V_{\mathrm{g}}$. Then, as some area becomes filled up by the latest emerging spots, the area as a whole becomes strongly transparent, giving rise to a transparency peak like those in Fig. 1b. The reverse sequence describes the falling edge of the strong transparency peak. An example of an area becoming strongly transparent as the last spot completes the merging is shown on a gray scale in Fig. 2. The transparency signal, acquired over an area of $5 \times 5 \mu \mathrm{m}^{2}$, is shown for three $V_{\mathrm{g}}$ voltages, a total electron density change of 2.5 electron $/ \mu \mathrm{m}^{-2}$. The only significant change to the shape of the transparent regions is the addition of a transparent (darker) spot centered at around $(35,25)$ and subtraction of the spot at $(30,2)$, which is not adjoining the main transparent area. However, the whole area becomes significantly more transparent, while mostly preserving its shape.

The tendency of larger blobs to display a stronger transparency can be understood on the following model. We assume that the blob is made up of multiple isolated (at $70 \mathrm{~Hz}$.) islands. The potential modulation induced on an island is proportional to $C_{\mathrm{bg}} / C_{\Sigma}$ where $C_{\mathrm{bg}}$ is the island-backgate capacitance and $C_{\Sigma}$ is the total capacitance of the island. $C_{\Sigma}$ is determined mainly by the distance between the island and the compressible regions. The maximal potential amplitude and, therefore, the maximal transparency occur farthest from the compressible area. The larger the blob, the stronger the transparency signal in its center. The amplitude of AC potential drops toward the edges of the blobs as the screening compressible area becomes closer. All this is in agreement with the experimental observations (Fig. 2).

Quite different patterns of transparency are observed in regions with significantly larger built-in potential gradients. Representative transparency curves are shown in Fig. 3a. There is no out-of-phase signal exceeding the noise floor all over the range, indicating that the charging is in equilibrium at $70 \mathrm{~Hz}$. The typical local density gradient in this region is $\sim 1 \times 10^{14} \mathrm{~cm}^{-3}, 10 \times$ steeper than for the region shown in Fig. 1b. The transparency signal, which is 

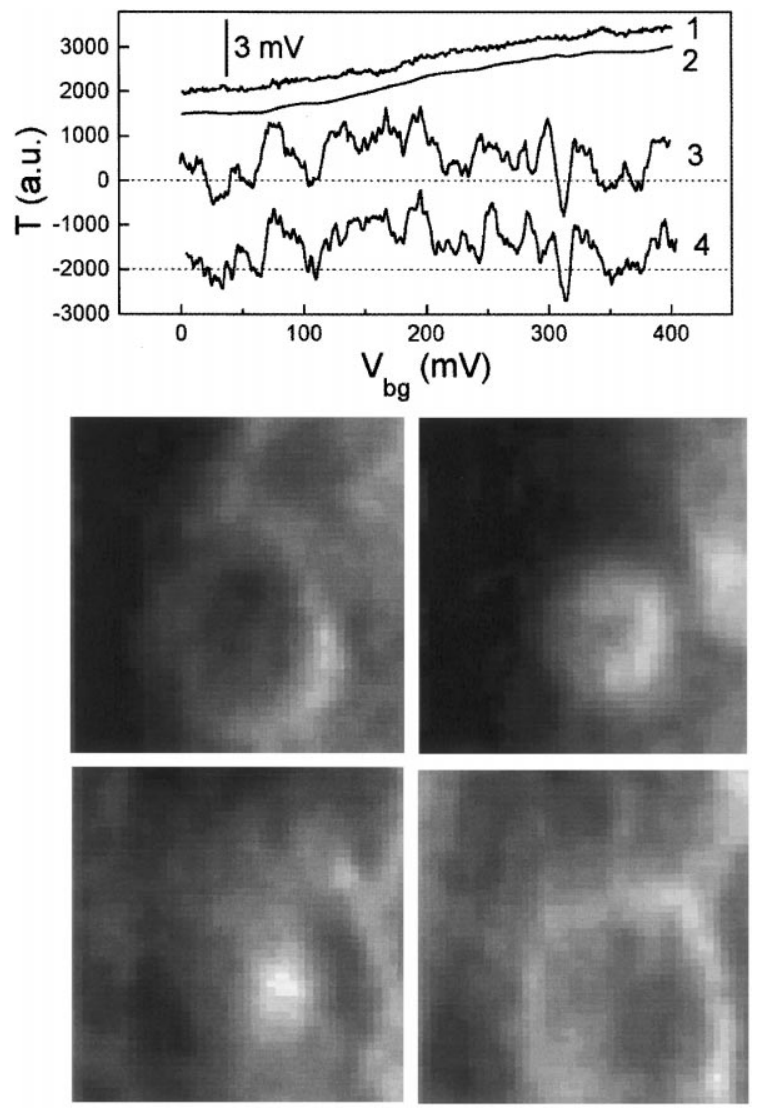

Fig. 3. (a) DC (1) and AC in-phase $(3,4)$ signals as a function of $V_{\mathrm{g}}$ measured at arbitrary location in a region of weak transparency at $v=2$. Curves (3) and (4) are taken sweeping $V_{\mathrm{g}}$ forth and back to illustrate the reproducibility. The integrated AC signal (2) closely reproduces the measured DC signal (1). (b) Spatial images of the in-phase AC signal over $0.45 \times 0.45 \mu \mathrm{m}^{2}$ area of weak transparency taken at four different densities. $V_{\mathrm{g}}$ is changed by 15 $\mathrm{mV}$ between images which adds on average $\sim 0.4$ electrons to the total area of the frame. White denotes zero or slightly negative transparency.

$\sim 5 \times$ weaker than in the previous example, displays multiple oscillations with peaks separated by $30-40$ $\mathrm{mV}$ in $V_{\mathrm{g}}$. The oscillation pattern is generally reproducible as illustrated by the traces (3) and (4) but can slowly vary with time. In places, the transparency oscillations reach definite, reproducible negative values. The DC signals show corresponding step structure, but these are more often obscured by noise.

The corresponding spatial maps are complex. However, when the oscillations with density are well defined, we generally find relatively simple patterns whose features move about with density, giving rise to the oscillations. An example of such maps is shown in Fig. $3 b$. The scanned area of $0.45 \times 0.45 \mu \mathrm{m}^{2}$ is shown for four values of $V_{\mathrm{g}}$ changed by $15 \mathrm{mV}$ between the panels, nominally an addition of 0.4 electrons over the panel area. There are clearly seen ring-like structures contracting to a common center. The transparency signals on the rings are comparatively small, and sometimes negative, while those in the center of the rings (dark regions) are larger and positive. For this particular place we observed two full periods of rings oscillation as the density is swept. In the general case, the patterns are much more complicated. We often observe several circular objects placed close one to another. As the objects expand and collide, the overall pattern evolves rapidly, losing self-similarity quickly as a function of density. We do not see any correlation between the extrema of the surface potential and the centers of the rings.

The multiple oscillations of the transparency are reminiscent of single-electron charging effects in quantum dots [9]. There the analogous Coulombblockade charging behavior in quantum dots would be saw-tooth changes of DC potential. Other experimental facts also parallel the single-electron blockade behavior. First, the quasi-period of the rings against the density increment approximately corresponds to the addition of a single-electron charge over the maximal correlated area of the structure. Second, as noted above, the peaks often overshoot the level of zero yielding negative transparency, as is seen in the capacitance measurements under Coulomb blockade conditions [10].

In some manner the contraction of a ring through one cycle adds one electron to the immediate area, but just how is not clear. A model in which the electron is being transferred into a fixed potential energy minimum created by disorder does not provide the observed continuous spatial variation of electrical potential. Also, the screening is weak. It is broadly accepted that the density of the carriers able to move freely and to participate in the screening is determined by the deviation from the integral filling [11]. This density is less than $3 \times 10^{9} \mathrm{~cm}^{-2}$ yielding the average distance between the free carriers of $>200 \mathrm{~nm}$. This distance is comparable to the typical sizes of the ring patterns. It is not clear 
how the continuous variation of the potential of the ring evolution can be provided by so few screening carriers.

In analogy with the oscillating regions, we believe that the strongly transparent regions with incomplete charging are built up from single-localized electron states. The formation of the blobs by merging small, slightly transparent spots supports this idea. But unlike the weak transparency case, the electron states inside blobs are localized far apart and do not overlap with each other or with the extended (compressible) states.

The distinction of the regions with either strong or weak transparency with respect to the built-in potential gradient can be understood qualitatively. It is predicted [12] and observed experimentally [6] that the contour lines with integer-filling factors widen into strips. This depletes the number of carriers able to screen the disorder potential within the strip. The macroscopic electrostatic model yields the strip width of $\sim 1.2 \mu \mathrm{m}$ for the gradient of $\sim 1 \times 10^{13} \mathrm{~cm}^{-3}$ typical for the regions occupied by the strongly transparent blobs. The screening of the disorder potential by the compressible states $1 \mu \mathrm{m}$ apart is not very efficient and electrons are strongly localized. With the gradient of $\sim 1 \times 10^{14} \mathrm{~cm}^{-3}$, the strip width is $\sim 400 \mathrm{~nm}$, improving the screening. This reduces effective disorder strength allowing for significant correlation effects.
In summary, the experiment described here has shown strong evidence for single-electron localized states in the QH regime with the image contrast originating either from the local resistance or from the local charging blockade.

\section{References}

[1] R.E. Prange, S.M. Girvin (Ed.), The Quantum Hall Effect, Springer, Berlin, 1990.

[2] R.B. Laughlin, Phys. Rev. B 23 (1981) 5632.

[3] M.J. Yoo et al., Science 276 (1997) 579.

[4] S.H. Tessmer et al., Nature 392 (1998) 51.

[5] K.L. McCormick et al., Phys. Rev. B 59 (1999) 4654.

[6] A. Yacoby et al., Solid State Commun. 111 (1999) 1.

[7] T.A. Fulton, G.J. Dolan, Phys. Rev. Lett. 59 (1987) 109.

[8] T.P. Smith et al., Phys. Rev. B 32 (1985) 2696.

[9] M.A. Kastner, Phys. Today 46 (1993) 24.

[10] D. Berman et al., Phys. Rev. Lett. 82 (1999) 161.

[11] A.L. Efros, Solid State Commun. 67 (1988) 1019.

[12] D.B. Chklovskii, B.I. Shklovskii, L.I. Glazman, Phys. Rev. B 46 (1992) 4026. 